\title{
The Pattern of Cardiac Arrhythmias in Acute ST Elevated Myocardial Infarction and their in-hospital Outcome
}

\author{
MOHAMMAD KHURSHADUL ALAM, MANZOOR MAHMOOD, DIPAL KRISHNAADHIKARY, FAKHRUL ISLAM \\ KHALED, MSI TIPU CHOWDHURY, AMANAT HASAN, SAMI NAZRUL ISLAM, MD. ASHRAF UDDIN SULTAN, \\ SAJAL KRISHNA BANERJEE \\ Department of Cardiology, Bangabandhu Sheikh Mujib Medical University, Dhaka,Bangladesh.
}

Address of Correspondence: Dr. Muhammad Khurshadul Alam, Department of Cardiology,Bangabandhu Sheikh MujibMedical University,Dhaka,Bangladesh. Email: dr.khurshdalam@gmail.com

\begin{abstract}
:
Background: Acute myocardial infarction (AMI) is a major cause of death worldwide with arrhythmia being the most common determinant in the post-infarction period. Identification and management of arrhythmias at an early period of acute MI has both short term and long term significance. Objective: The aim of the study is to evaluate the pattern of arrhythmias in acute STEMI in the first 48 hours of hospitalization and their inhospital outcome. Methods: A total of 50 patients with acute STEMI were included in the study after considering the inclusion and exclusion criteria. The patients were observed for the first 48 hours of hospitalization for detection of arrhythmia with baseline ECG at admission and continuous cardiac monitoring in the CCU. The pattern of the arrhythmias during this period \& their in-hospital outcome were recorded in predesigned structured data collection sheet.Result: The mean age was $53.38 \pm 10.22$ years ranging from 29 to 70 years. Most of the patients were male 42(84\%). Majority of the patients had anterior wall (anterior, antero-septal \& extensive anterior) myocardial infarction (54\%). Sinus tachycardia in isolation was the most common arrhythmia observed in $36.8 \%$ of patients followed by sinus bradycardia (22.8\%), ventricular tachycardia (19.3\%), ventricular ectopic (12.3\%), first degree AV block (5.3\%), complete heart block and atrial ectopic $1.7 \%$ each. Tachyarrhythmias were more common in anterior wall myocardial infarction, whereas bradyarrhythmias were more common in inferior wall myocardial infarction. Among studied patients, $72 \%$ had favourable outcome, followed by acute left ventricular failure 10\%, cardiogenic shock \& lengthening of hospital stay 8\% each and death 2\%.Conclusion: The commonest arrhythmias encountered were sinus tachycardia followed by sinus bradycardia, ventricular tachycardia, ventricular ectopic, AV block and atrial ectopic. The incidence of mortality was $2 \%$.
\end{abstract}

Keywords: Arrhythmia, Acute ST-segment elevated myocardial infarction, Outcome.

University Heart Journal 2020; 16(1): 16-21

Introduction:

Cardiovascular diseases (CVDs) are the leading causes of death globally.Despite decreasing mortality trends of coronary artery disease (CAD) in many developed countries, increasing number is noticed in developing countries. ${ }^{1}$ Acute myocardial infarction (AMI) is myocardial necrosis in a clinical setting consistent with acute myocardial ischemia and detection of elevated values of cardiac biomarkers (troponin-I/ CK-MB) above the 99 th centile of the upper reference limit. ${ }^{2} \mathrm{~A}$ substantial number of patients with acute myocardial infarction have some cardiac rhythm abnormality and most cases cardiac conduction disturbance develop within 48 hours following infarct onset. Deaths most commonly occur within first few hours of acute myocardial infarctions. Early deaths are not related to the severity of infarct but observations from monitoring unit suggest that the mechanism in most of the cases is arrhythmias and cardiac asystole. Almost any rhythm disturbance can be associated with acute myocardial infarction, including bradyarrhythmias, supraventricular tachyarrhythmias, ventricular arrhythmias and atrioventricular block.

It is known that myocardial infarction leads to severe metabolic and electrophysiological changes that induce silent or symptomatic life-threatening arrhythmias. The most important assumption for key mechanism of arrhythmia in acute phase of coronary occlusion is microreentry due to inhomogeneity of electrical characteristics of ischemic myocardium. Cells of center of the ischemic zone have a relatively consistent increase in 
extracellular potassium concentration. Whereas, cells in the border zone between ischemic region and normal myocardium are only partially depolarized and consequently have action potentials with larger amplitude. Slowing of impulse conduction take place in noticeably depressed areas leading to arrhythmias. The cellular electrophysiological method for reperfusion arrhythmias emerge to include washout of different ions such as lactate $\&$ potassium and toxic metabolic substance that have accumulated in ischemic zone. ${ }^{3}$ Peri-infarction arrhythmias are related with in-and out-hospital outcome of the patients, there is no available data of arrhythmias in AMI patients of Bangladesh. This study was to document the pattern of arrhythmias in acute ST elevated MI within 48 hours of hospitalization and their in-hospital outcome among the population of Bangladesh.

\section{Methods:}

This observational study was conducted in Cardiology Department of BSMMU during July, to December, 2018. Patients who admitted with acute STEMI in department of cardiology, BSMMU were initially approached and included in this study considering the inclusion and exclusion criteria. A total of 50 patients were included in the study.Before inclusion of the patient, purpose and procedure of the study was discussed with the patients and informed written consent was taken from those who agreed to participate in the study.

\section{Inclusion Criteria:}

1. Patients 18 years of age or above.

2. Patients with acute STEMI.

3. Patient who has given informed written consent.

\section{Exclusion Criteria:}

1. Patients less than 18 years of age.

2. NSTEMI.

3. Patients with documented arrhythmia before the episode of acute MI.

4. Patients with previous history of MI.

5. Patients with structural and valvular heart disease.

A detailed history with particular emphasis on the risk factors were noted and general physical along with thorough systemic examination was done. The patients were observed for the first 48 hours of hospitalization for detection of arrhythmia with baseline ECG at admission and continuous cardiac monitoring in the CCU.The pattern of the arrhythmias during this period $\&$ their in-hospital outcome were recorded in predesigned structured data collection sheet. After editing data analysis was carried out by using the Statistical Package for Social Science (SPSS) version 20.0 windows software. Continuous data were expressed as mean $\pm \mathrm{SD}$. Categorical data were expressed as frequency and percentages. Categorical data were analyzed by Chi-Square test. $\mathrm{P}$ value of less than 0.05 was considered significant. The study was performed according to the guidelines of the Helsinki Declaration and was approved by the University ethical committee

\section{Results:}

A total of 50 patients were studied. Maximum number of patients were from age group 51 to 60 years $(38 \%)$. The mean age was $53.38 \pm 10.22$ years ranging from 29 to 70 years. Most of the patients were male 42(84\%). All of the female were house wife. Highest number of patients were businessman 15(30\%) (Table 1).

$76 \%$ of the patients had history of smoking followed by hypertension $64 \%$, DM 40\%, dyslipidemia 38\%, overweight $34 \%$ and family history of CAD 18\% (Table 2 ).

Table-I

Socio-demographic variables

\begin{tabular}{llcc}
\hline Socio-Demographic Variables & & Frequency & Percentage $(\%)$ \\
\hline Age Groups & $<40$ Years & 06 & 12.0 \\
& $41-50$ Years & 13 & 26.0 \\
& $51-60$ Years & 19 & 38.0 \\
Sex & $>60$ Years & 12 & 24.0 \\
& Male & 42 & 84.0 \\
Occupation & Female & 08 & 16.0 \\
& Businessman & 15 & 30.0 \\
& House Wife & 08 & 16.0 \\
& Service Holder & 05 & 10.0 \\
Total & Retired & 09 & 18.0 \\
\hline
\end{tabular}


Table-II

Distribution of risk factors

\begin{tabular}{|c|c|c|c|}
\hline Risk Factors & & Frequency & Percentage $(\%)$ \\
\hline \multirow[t]{4}{*}{ BMI Status } & Normal Weight & 26 & 52.0 \\
\hline & Under Weight & 02 & 4.0 \\
\hline & Over Weight & 17 & 34.0 \\
\hline & Obese & 05 & 10.0 \\
\hline \multirow[t]{2}{*}{ Family H/o CAD } & Present & 09 & 18.0 \\
\hline & Absent & 41 & 82.0 \\
\hline \multirow[t]{2}{*}{ Diabetes Mellitus } & Diabetic & 20 & 40.0 \\
\hline & Non Diabetic & 30 & 60.0 \\
\hline \multirow[t]{2}{*}{ Hypertension } & Hypertensive & 32 & 64.0 \\
\hline & Non Hypertensive & 18 & 36.0 \\
\hline \multirow{2}{*}{ Smoking Status } & Smoker & 38 & 76.0 \\
\hline & Non Smoker & 12 & 24.0 \\
\hline \multirow[t]{2}{*}{ Dyslipidaemia } & Present & 19 & 38.0 \\
\hline & Absent & 31 & 62.0 \\
\hline Total & & 50 & 100.0 \\
\hline
\end{tabular}

$46 \%$ of the patients had inferior MI followed by anterior MI 36\%, antero-septal MI16\% and extensive anterior MI $2 \%$ (Table 3 ).

Among the 50 patients 44 patients had developed single arrhythmia, 5 developed double arrhythmias and one patient developed triple arrhythmias in the first 48 hours of hospitalization. Sinus tachycardia in isolation was the most common arrhythmia observed in $36.8 \%$ of patients followed by sinus bradycardia $22.8 \%$, ventricular tachycardia $19.3 \%$, ventricular ectopic $12.3 \%$,first degree AV block 5.3\%, complete heart block and atrial ectopic $1.7 \%$ each. Overall tachyarrhythmias (sinus \& ventricular tachycardia) occurred in $56.1 \%$ patients and bradyarrhythmias (sinus bradycardia \& complete heart block) in $24.5 \%$. Overall incidence of ventricular arrhythmias (ventricular tachycardia \& ventricular ectopic) was $31.6 \%$ \& AV blocks(first degree \& complete heart block ) was 7\% (Table 4).

Majority of arrhythmias (71.9\%) occurred $<12$ hours of hospitalization. Progressively arrhythmia occurrence decreased with time (Table 5).

$72 \%$ patients had favorable outcome, followed by acute left ventricular failure $10 \%$, cardiogenic shock \& lengthening of hospital stay $8 \%$ each and death $2 \%$ (Table 6).

Tachyarrhythmias (sinus \& ventricular tachycardia) were more common in anterior wall(anterior, antero-septal\& extensive anterior) myocardial infarction, whereas bradyarrhythmias (sinus bradycardia \& AV block) were more commonin inferior wall myocardial infarction. This association between anatomical site of myocardial infarction and types of cardiac arrhythmias is statistically very highly significant $(\mathrm{P}=0.000$ ) (Table 7).

Table-III

Distribution of anatomical sites of MI

\begin{tabular}{lcc}
\hline Site of MI & Frequency & Percentage (\%) \\
\hline Inferior & 23 & 46.0 \\
Anterior & 18 & 36.0 \\
Antero-septal & 08 & 16.0 \\
Extensive Anterior & 01 & 2.0 \\
\hline Total & 50 & 100.0 \\
\hline
\end{tabular}

Table-IV

Different types of arrhythmias documented in study subjects $(n=57)$

\begin{tabular}{lcc}
\hline Cardiac Arrhythmia & Frequency & Percentage (\%) \\
\hline Sinus Tachycardia & 21 & 36.8 \\
Sinus Bradycardia & 13 & 22.8 \\
Ventricular Tachycardia & 11 & 19.3 \\
Ventricular Ectopic & 07 & 12.3 \\
First Degree AV Block & 03 & 5.3 \\
Complete Heart Block & 01 & 1.7 \\
Atrial Ectopic & 01 & 1.7 \\
\hline Total & 57 & 100.0 \\
\hline
\end{tabular}


Table-V

Time of appearance of individual arrhythmias after hospitalization $(n=57)$

\begin{tabular}{lcc}
\hline $\begin{array}{l}\text { Time of Appearance of } \\
\text { Cardiac Arrhythmia }\end{array}$ & Frequency & Percentage (\%) \\
\hline$<12$ Hours & 41 & 71.9 \\
$12-24$ Hours & 09 & 15.8 \\
$24-48$ Hours & 07 & 12.3 \\
\hline Total & 57 & 100.0 \\
\hline
\end{tabular}

Table-VI

In-hospital outcomes

\begin{tabular}{lcc}
\hline Outcome & Frequency & Percentage (\%) \\
\hline Death & 01 & 2.0 \\
Cardiogenic Shock & 04 & 8.0 \\
Acute Left Ventricular Failure & 05 & 10.0 \\
Lengthening of Hospital Stay & 04 & 8.0 \\
Favorable Outcome & 36 & 72.0 \\
\hline Total & 50 & 100.0 \\
\hline
\end{tabular}

Table-VII

Association between anatomical sites of myocardial infarction and types of cardiac arrhythmias $(n=49)$

\begin{tabular}{lccc}
\hline & \multicolumn{3}{c}{ Cardiac Arrhythmia } \\
& Tachyarrhythmia & Bradyarrhythmia & Total \\
\hline Anterior & 23 & 01 & 24 \\
Inferior & 09 & 16 & 25 \\
\hline Total & 32 & 17 & 49 \\
\hline
\end{tabular}

Chi-square $\left(X^{2}\right)=19.348 ; P=0.000$ (Very Highly Significant)

\section{Discussion:}

The age distribution in this present study ranged from 29 to 70 years with maximum number of patients in the age group 51 to 60 years. This is comparable with findings of Patil at el., ${ }^{4}$ who found the maximum number of patients in this similar age group. The mean age in this present study was $53.38 \pm 10.22$ years, which is comparable with the studies of Patil, B.M., ${ }^{5} \&$ Chowdhury et al., ${ }^{6}$ who found mean age of the patients $53.61 \pm 12.43$ years \& $53 \pm 10$ years respectively.

Among the study subjects $42(84 \%)$ were male and $8(16 \%)$ were female. Similar male preponderance was found in almost all studies on arrhythmia in AMI. Patil at el., ${ }^{4}$ observed $77.50 \%$ male compared to $22.50 \%$ female \& Rajhans et al., ${ }^{7}$ observed $70 \%$ male compared to $30 \%$ female. As females are given less attention and access to the health care facilities is limited particularly in low socioeconomic population like our country may contribute to this male predominance.

Risk factors analysis revealed that $76 \%$ of the patients had history of smoking. This is comparable with study of Patil, B.M., ${ }^{5}$ who observed $70 \%$ of cases were smoker. Hypertension (64\%) was the second commonest risk factor after smoking . Other risk factors include DM (40\%), dyslipidemia (38\%), overweight (34\%) and family history of CAD (18\%). According to Rajhans et al., ${ }^{7}$ incidence of hypertension and diabetes were $66 \%$ and $54 \%$ respectively, which is very close to findings of this study. Patil, B.M., ${ }^{5}$ found $36 \%$ of cases were dyslipidaemic, which is comparable with the present study.

In this study, majority of the patients had anterior wall ( anterior, antero-septal\& extensive anterior) myocardial infarction (54\%). This is comparable with the study of Rajhans et al., ${ }^{7}$ where overall incidence of anterior wall was higher $(56 \%)$ than inferior wall which was $44 \%$. Similarly according to Mhatre et al. ${ }^{8}$ incidence of anterior wall myocardial infarction (58\%).

In this study sinus tachycardia was the most common arrhythmia observed in $36.8 \%$ cases. According to the study by Patil, B.M., ${ }^{5}$ commonest arrhythmia was sinus tachycardia (40\%), which is comparable with the current study. In other studies by Maturaju, N.\&Chandrashekhar, H.M., ${ }^{9}$ and Sinha at el., ${ }^{10}$ found sinus tachycardia were the commonest arrhythmias seen in $30 \%$ \& 31\% cases respectively. In the current study the second commonest arrhythmia was sinus bradycardia $22.8 \%$, which is comparable with other studies by Nagabhushana et al., ${ }^{11}$ and Rathod at al., ${ }^{12}$ who found sinus bradycardia $22 \%$ $\& 19 \%$ respectively . Ventricular tachycardia was 19.3\%, which is comparable with other studies of Maturaju, N. \&Chandrashekhar, H.M., ${ }^{9}$ and Sinha at el., ${ }^{10}$ who found ventricular tachycardia $20 \% \& 25 \%$ respectively. In this study ventricular ectopic was $12.3 \%$. This is in conformity with study by Chiwhane at el., ${ }^{13}$ who found ventricular ectopic in $17 \%$ patients.In this study overall incidence of AV blocks was 7\%, compared to other studies like Marangmei et al., ${ }^{14}$ who found AV blocks almost double i.e. $15 \%$. This disparity in incidence may be attributed to the small sample size in the current study. In the present study first degree AV block was 5.3\%. This is comparable to study byRathod at al., ${ }^{12}$ andNagabhushana et al., ${ }^{11}$ who observed first degree AV block 4\% \& 3\% respectively.

In this study majority of arrhythmias (71.9\%) occurred within 12 hours of hospitalization. Similar findings were also observed by Patil, B.M., ${ }^{5}$ where majority of arrhythmias occurred within 12 hours of hospitalization. 
In the current study $87.7 \%$ arrhythmias occurred within 24 hours of hospitalization. This is in conformity with study by Patil at el., ${ }^{4}$ where majority of arrhythmias (90\%) occurred during the first 24 hours hospitalization.

$72 \%$ of patients had favorable outcome, Similar outcome was found in almost all studies on arrhythmia in AMI. In the present study $10 \%$ patients developed acute left ventricular failure and $8 \%$ cardiogenic shock which are comparable with study of Sinhaet al., ${ }^{10}$ who found acute left ventricular failure and cardiogenic shock in $17 \%$ \& $11 \%$ patients respectively. This slight disparity in incidence can be attributed to the small sample size in the present study. Tendency to go in shock was more common in CHB and VT, which is also well supported by study of Sinhaet al., ${ }^{10}$.

In this study there was one death $(2 \%)$ that occurred in a patient with anterior MI who developed VT within 12 hours of hospitalization. According to the study of Patil, B.M., ${ }^{5}$ the overall incidence of mortality was $15 \%$, where majority of mortality occurred within 24 hours of hospitalization \& most of the deaths occurred in VT \& CHB. Advanced quality of CCU \& ICU management of STEMI in University Cardiac Center, BSMMU and small sample size may have contributed to lower in-hospital mortality in this study.

In the current study tachyarrhythmias (sinus tachycardia and ventricular tachycardia) were more common in anterior wall (anterior, antero-septal\& extensive anterior) myocardial infarction, whereas bradyarrhythmias( sinusbradycardia\& AV block ) were more common in inferior wall myocardial infarction. This association between anatomical site of myocardial infarction and types of cardiac arrhythmias is statistically very highly significant $(p=0.000)$. Similar association between anatomical site of myocardial infarction and types of cardiac arrhythmias were observed by Rathod at al., ${ }^{12}$ and Marangmei et al. ${ }^{14}$

Though the findings in this study are mostly in agreement with previous studies on arrhythmias in acute STEMI, some findings were insignificant or in consistent. This may be due to the following limitations:

- Single centere study.

- Result of the study might be influenced by relatively small ersamplesize.

- Sampling method was purposive, so there was risk of selectionbias.

\section{Conclusion:}

The commonest arrhythmias encountered were sinus tachycardia followed by sinus bradycardia, ventricular tachycardia, ventricular ectopic, AV block and atrial ectopic. Most of arrhythmias developed within 12 hours of hospitalization. Tachyarrhythmias were more common in anterior wall myocardial infarction, whereas bradyarrhythmias were more common in inferior wall myocardial infarction. The incidence of mortality was $2 \%$.

\section{Conflict of interest:}

Authors has no conflict of interest.

\section{Acknowledgements:}

This research was a postgraduate thesis. The authors would like to thank all the patients who have participated in this randomized controlled trial. Financial support was obtained from the research council of the Bangabandhu Sheikh Mujib Medical University, Dhaka.

\section{Reference:}

1. Bhatnagar, P., Wickramasinghe, K., Williams, J., Rayner, M. and Townsend, N., 2015, 'The epidemiology of cardiovascular disease in the UK 2014; Heart, 101(15): 1182-1189.

2. Thygesen, K., Alpert, J. S., Jaffe, A. S., Somoons, M., Chaitman, B. R. and White, H. D., 2018, '2018 ESC/ACC/ AHA/WHF Fourth Universal Definition of MI', Circulation. Vol.138, p. e4-34.

3. Antman, EM., 2008, 'ST elevation myocardial infarction: Management', in Zipes, D.P., Libby, P., Bonow, A., Braunwald, E., Braunwald's Heart Disease, $8^{\text {th }}$ ed, Philadelphia, Saunders, pp. 1276-9.

4. Patil, P.R., Khatana, P. \&Patil, D.R. 'Incidence of cardiac arrhythmias in patients with acute myocardial infarction during the first 48 hours of the onset of chest pain', Int $J$ Adv Med. 2017;4(4):1144-49.

5. Patil, B.M. 'Study on site of acute myocardial infarction associated with arrhythmias and outcome', International Journal of Advances in Medicine, 2015;2(1):21-25.

6. Chowdhury, R., Alam, D.S., Fakir, I.I., Adnan S.D., Naheed, A., Tasmin, I. et al. 'The Bangladesh Risk of Acute Vascular Events (BRAVE) Study', European Journal of Epidemiology, 2015;30(7):577-587.

7. Rajhans, R. \& Narayanan, M. 'Assessment of arrhythmias in 50 patients of ST-elevation myocardial infarction after thrombolysis: a 24 hour Holter study', Int J Adv Med, 2017;4(3):734-40.

8. Mhatre, M.A., Sirur, F.M., Rajpal, D.R. \& Shah, M.R. 'A clinical study of arrhythmias associated with acute myocardial infarction and thrombolysis', International Journal of Research in Medical Sciences, 2017;5(1):335-343.

9. Maturaju, N. \& Chandrashekhar, H.M. 'The pattern of arrhythmias during first 48 hours of acute myocardial infarction', International Journal of Medical Research, 2016;1(4):38-40. 
10. Sinha, R.P., Agrawal, D. \& Jain, A. 'Incidence of various arrhythmias and its prognosis in different type of ST segment elevation myocardial infarction in first 72 hours',J. Evid. Based Med. Healthc. 2018;5(39):2772-77.

11. Nagabhushana, S., Ranjithkumar, G.K., Ranganatha, M. \&Virupakshappa. 'Study of Arrhythmias in Acute Myocardial Infarction ', International Journal of Medical Research and Review, 2015;3(7):682-88.

12. Rathod,S., Parmar, P., Rathod, G.B. \& Parikh, A. 'Study of various cardiac arrhythmias in patients of acute myocardial infarction ', International Archives of Integrated Medicine, 2014; 1(4):32-41.

13. Chiwhane, A. \& Pradeep. 'Study of Rhythm Disturbances in Acute Myocardial Infarction', Journal of The Association of Physician of India, 2018;66:54-58.

14. Marangmei, L., Singh, S.K., Devi, K.B., Raut, S.S., Chongtham, D.S. \& Singh, K.B. 'Profile of cardiac arrhythmia in acute myocardial infarction patients within 48 hours of admission: A hospital based study at RIMS Imphala', Jounal of Medical Society, 2014;28(3):175-78. 